\title{
Mechanics of Energy Materials
}

\author{
S. Xia ${ }^{1} \cdot$ P. Guduru ${ }^{2} \cdot$ H. Sodano ${ }^{3}$ \\ Published online: 15 March 2018 \\ (C) Society for Experimental Mechanics 2018
}

Advanced energy materials hold the key to fundamental advances in the conversion, storage, and harvesting of energy for a broad range of consumer electronics, automotive, aerospace, and defense applications. The successful development and deployment of these materials rely critically on a fundamental understanding of strongly coupled multiphysics phenomena. Mechanics of energy materials has emerged as a rapidly growing area of research that has significant technological implications in improving the efficiency, reliability, and sustainability of energy infrastructure. This Special Issue aims to exchange the latest research advances in this highly interdisciplinary area, with a focus on experimental and integrated computational/ experimental investigations from the mechanics and materials prospectives.

The eleven papers in this Special Issue are classified into four groups: (1) in situ and ex situ characterization of stress, deformation, and mechanical degradation in electrochemically active energy storage materials; (2) characterization of coupling phenomena between mechanical and electrochemical processes in rechargeable battery electrode materials; (3) mechanical behavior of lithium-ion battery (LIB) cells under quasi-static and dynamic loading; and (4) mechanics of piezoelectric materials for energy harvesting from mechanical/ vibration sources. In the following, we provide a summary of the main contribution of each paper.

High-capacity LIB anode materials, such as silicon ( $\mathrm{Si}$ ), germanium $(\mathrm{Ge})$, and tin $(\mathrm{Sn})$, are prone to fracture due to lithiation-induced volumetric expansion. A great deal of recent

\author{
S. Xia \\ sxia30@mail.gatech.edu \\ P. Guduru \\ Pradeep_Guduru@brown.edu \\ H. Sodano \\ hsodano@umich.edu \\ Georgia Institute of Technology, Atlanta, GA, USA \\ Brown University, Providence, RI, USA \\ University of Michigan, Ann Arbor, MI, USA
}

work has shown the promise of oxide coatings in preventing or mitigating electrode fracture. However, a mechanistic understanding of how these oxide coatings work is still largely missing. The paper by Rakshit et al. presents the first mechanical characterization of an oxide coating subjected to lithiation/ delithiation cycling. A multi-beam optical sensor (MOS) setup was employed for the in situ stress measurement of a $\mathrm{SiO}_{2}$ thin film cycled against a lithium foil. A large compressive biaxial stress of $\sim 3 \mathrm{GPa}$ was measured during lithiation. Upon delithiation, the stress became tensile with a peak value of $0.7 \mathrm{GPa}$. The measured stress response was fed to a finite element model to provide a mechanistic explanation for the improved cyclic performance of $\mathrm{Si} / \mathrm{SiO}_{2}$ nanotubes. These findings are of fundamental importance, both in developing constitutive models of oxide coating materials and in designing mechanically and chemically stable coating-based battery electrodes.

The paper by $\mathrm{Xu}$ et al. is concerned with the mechanical degradation of lithium nickel manganese cobalt (NMC) oxide, a high-capacity cathode material that is increasingly used in electric vehicle (EV) applications. The microstructural evolution of NMC composite electrodes during electrochemical cycling was analyzed with scanning electron microscopy (SEM). The SEM analysis was combined with electrochemical impedance spectroscopy (EIS) to study the effects of charging rate on the disintegration of NMC particles. The experimental results were supplemented by computational cohesive zone modeling to understand the deformation and damage accumulation mechanisms in NMC electrodes.

Full-field deformation measurement techniques are now playing an indispensable role in mechanical property characterization. When coupled with electrochemical characterization tools, they can provide valuable insights into energy storage materials. In the work reported by Capraz et al., the digital image correlation (DIC) method was employed for the in situ deformation analysis of an electrochemically cycled lithium manganese oxide (LMO) thin-film cathode. Despite many advantages over other LIB cathode materials, LMO suffers from poor interface stability, manganese dissolution in liquid electrolyte, and electrode particle cracking. Capraz et al. revealed the existence of irreversible strain in LMO during the first 
delithiation/lithiation cycle, and correlated it with the dissolution of native surface layer and the formation of cathodeelectrolyte interface (CEI) layer. A capacity fade was observed during subsequent cycling and was found to scale linearly with the maximum strain developed in the cathode. These experimental findings suggest a viable means for improving the electrochemical performance of LMO by controlling its mechanical response.

The work of Gonzalez et al. represents the first application of high-resolution digital volume correlation (DVC) to composite battery electrodes. By combining three-dimensional (3D) imaging and DVC, the authors performed full-field deformation analysis of a graphite composite electrode. The graphite electrode was lithiated in a specially designed battery cell that allowed for in situ X-ray scanning. The electrode microstructures were reconstructed using computed tomography and processed through DVC analysis for quantitative 3D strain measurement. The electrode demonstrated negligible shear strain and relatively uniform volumetric expansion, which was about $75 \%$ of the theoretical value.

Faisal et al. applied neutron diffraction to measure residual strains in molybdenum carbide $\left(\mathrm{Mo}_{2} \mathrm{C}\right)$-based anode materials with various metal oxide precursors. $\mathrm{Mo}_{2} \mathrm{C}$-based anode materials possess higher mechanical strength and better thermal and wear resistance than other anode materials for use in solid oxide fuel cells (SOFCs). Owing to its non-destructive nature and high beam penetration, neutron diffraction was successfully used to provide through-thickness strain profiles of three different $\mathrm{Mo}_{2} \mathrm{C}$-based anode coatings. The neutron diffraction results were supplemented with X-ray diffraction, nanoindentation and SEM measurements for a detailed understanding of the structure-property relationship in $\mathrm{Mo}_{2} \mathrm{C}$-based anodes.

Stress-diffusion coupling in a battery electrode material is traditionally thought to adversely affect the electrochemical performance of the material. Schiffer and Arnold took a new perspective to harness stress-diffusion coupling for lowfrequency mechanical energy harvesting. They demonstrated that LIB electrode materials could be used to convert mechanical energy into electrical energy. This conversion process was experimentally characterized and theoretically modelled to gain an understanding of the efficiency of energy conversion. Their work provides a baseline for piezo-electrochemical energy harvesting applications.

Papakyriakou et al. combined nanoindentation with chemomechanical computational modeling to investigate the stressdiffusion coupling in lithiated $\mathrm{Ge}$ anode. The time-dependent creep behavior of lithiated Ge thin films was characterized using depth-sensing nanoindentation in an inert environment. Concurrently, a continuum finite element model was developed and used to elucidate the experimentally measured creep response. It was found that the nanoindentation creep behavior could be well rationalized by a stress-induced diffusion mechanism. The stress-diffusion coupling coefficient and diffusivity of lithium in Ge were determined by quantitatively comparing the experimental and computational results. This integrative experimental and computational study provides fundamental insights into the chemo-mechanical coupling process in largedeformation rechargeable battery electrodes.

The increasing use of LIBs in EVs has raised serious safety concerns in the case of mechanical abuse. This Special Issue has two papers devoted to the investigation of the macroscopic mechanical response of LIBs at the battery cell level. Tsutsui et al. studied the compressive mechanical behavior of commercial cylindrical LIB cells at various deformation rates and states of charge (SOCs). They showed that the mechanical and electrochemical responses of the cells, such as stiffness, critical failure load, and voltage change, were greatly influenced by the combination of SOC and deformation rate. $\mathrm{Xu}$ et al. also examined the mechanical integrity of LIBs, with a focus on the coupling effect of SOC and state of health $(\mathrm{SOH})$ under quasi-static loading conditions. Three types of cylindrical cells with different battery chemistries were used in a comparative study to investigate the SOC- and SOH-dependent thermal and mechanical properties of the batteries. These experimental studies are highly informative for designing crash-safe EV batteries and multifunctional batteries for both structural load bearing and energy storage.

Dielectrics are important energy generation and storage materials in the form of piezoelectrics and electrostatic capacitors. Xing, Radovic, and Muliana studied the dielectric and elastic behavior of nanocomposites consisting of barium titanate $(\mathrm{BaTiO} 3)$ and silver $(\mathrm{Ag})$ constituents. The authors used two micromechanics models describing the detailed composite microstructures to predict the bulk material behavior, and validated their predictions through experimental testing. The first model is based on two-dimensional (2D) images of realistic microstructures obtained by SEM, while the second model is based on randomly generated 3D microstructures with spherical particles. It is found that the constant electric potential gradient boundary condition gives an extremely large prediction which is an upper bound, while a uniform flux boundary leads to a lower bound. The experimentally measured relative permittivities were found to lie between the upper and lower bounds. It is hypothesized that the large fluctuations are a result of the high contrast in relative permittivity between silver and barium titanate.

Ionic actuators are a class of materials that use the accumulation of ions at a double layer to induce swelling and bending deformation in ionomers. Ionic actuators operate at low voltage and produce larges strains; however, the blocking force is often low. Sun et al. (Exp Mech 58:99-109, 2018) investigated a chitosan-based ionomer which allows for biocompatibility. Their analysis shows excellent electromechanical energy efficiency under a high electrical conductivity of the electrode layer, which enhanced the specific electromechanical energy up to $\sim 10 \%$. 
The papers in this Special Issue collectively provide a representative sample of current research in the field of mechanics of energy materials. As new energy materials are being developed and implemented, fundamental mechanics issues continue to emerge and call for intense interdisciplinary research. Advances in experimental mechanics of energy materials will enable the development of computational modeling tools with unprecedented predictive capabilities, and ultimately accelerate the design, optimization, and manufacturing of novel energy materials.

Finally, we would like to express our sincere gratitude to all of the contributing authors, the editorial team of Experimental Mechanics, and the Society for Experimental Mechanics. This Special Issue would not have been possible without their contributions and support. 